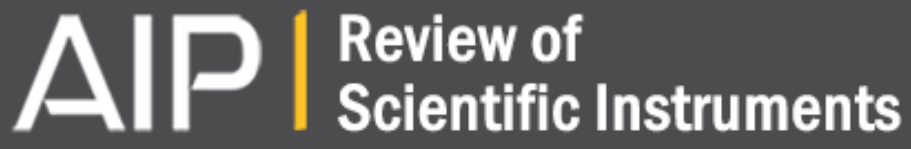

Model-independent quantitative measurement of nanomechanical oscillator vibrations using electron-microscope linescans

Huan Wang, J. C. Fenton, O. Chiatti, and P. A. Warburton

Citation: Review of Scientific Instruments 84, 075002 (2013); doi: 10.1063/1.4811740

View online: http://dx.doi.org/10.1063/1.4811740

View Table of Contents: http://scitation.aip.org/content/aip/journal/rsi/84/7?ver=pdfcov

Published by the AIP Publishing

\section{Articles you may be interested in}

Dynamic range of atomically thin vibrating nanomechanical resonators

Appl. Phys. Lett. 104, 103109 (2014); 10.1063/1.4868129

Uncertainty in least-squares fits to the thermal noise spectra of nanomechanical resonators with applications to the atomic force microscope

Rev. Sci. Instrum. 85, 025104 (2014); 10.1063/1.4864086

Nanomechanical tuning forks fabricated using focused-ion-beam chemical vapor deposition

J. Vac. Sci. Technol. B 30, 06FD03 (2012); 10.1116/1.4766315

Energy measurement in nonlinearly coupled nanomechanical modes

Appl. Phys. Lett. 98, 264106 (2011); 10.1063/1.3604797

Piezoelectric push-pull micropositioner for ballistic electron emission microscope

Rev. Sci. Instrum. 68, 3803 (1997); 10.1063/1.1148030
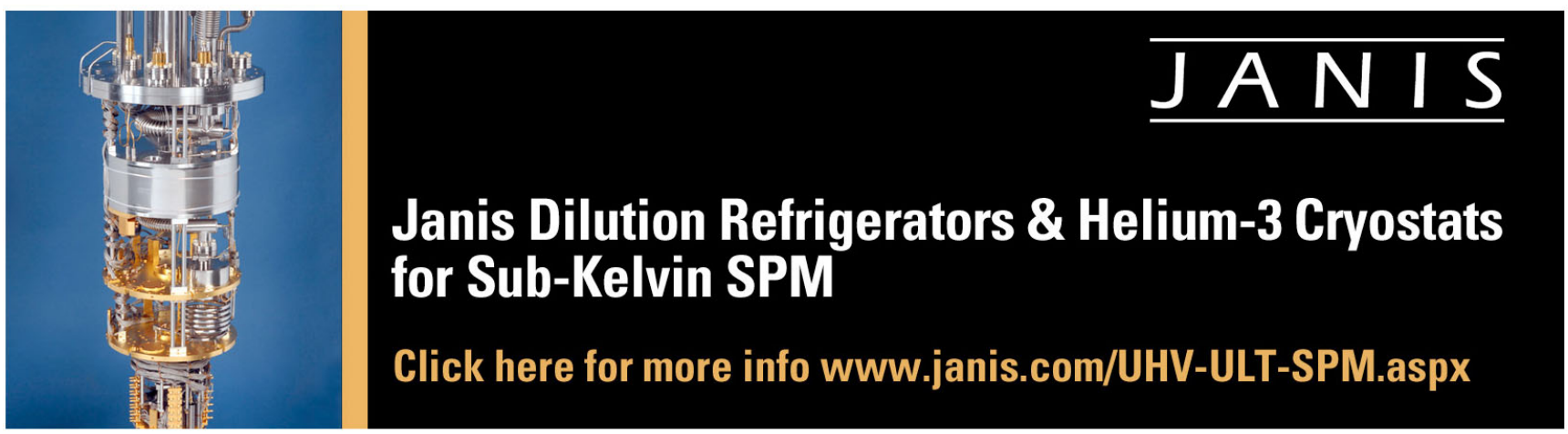


\title{
Model-independent quantitative measurement of nanomechanical oscillator vibrations using electron-microscope linescans
}

\author{
Huan Wang (王缓), ${ }^{1}$ J. C. Fenton, ${ }^{1}$ O. Chiatti, ${ }^{1}$,a) and P. A. Warburton ${ }^{1,2, b)}$ \\ ${ }^{1}$ London Centre for Nanotechnology, University College London, 17-19 Gordon Street, \\ London WC1H OAH, United Kingdom \\ ${ }^{2}$ Department of Electronic and Electrical Engineering, University College London, Torrington Place, \\ London WC1E 7JE, United Kingdom
}

(Received 11 February 2013; accepted 7 June 2013; published online 11 July 2013)

\begin{abstract}
Nanoscale mechanical resonators are highly sensitive devices and, therefore, for application as highly sensitive mass balances, they are potentially superior to micromachined cantilevers. The absolute measurement of nanoscale displacements of such resonators remains a challenge, however, since the optical signal reflected from a cantilever whose dimensions are sub-wavelength is at best very weak. We describe a technique for quantitative analysis and fitting of scanning-electron microscope (SEM) linescans across a cantilever resonator, involving deconvolution from the vibrating resonator profile using the stationary resonator profile. This enables determination of the absolute amplitude of nanomechanical cantilever oscillations even when the oscillation amplitude is much smaller than the cantilever width. This technique is independent of any model of secondary-electron emission from the resonator and is, therefore, applicable to resonators with arbitrary geometry and material inhomogeneity. We demonstrate the technique using focussed-ion-beam-deposited tungsten cantilevers of radius $\sim 60-170 \mathrm{~nm}$ inside a field-emission SEM, with excitation of the cantilever by a piezoelectric actuator allowing measurement of the full frequency response. Oscillation amplitudes approaching the size of the primary electron-beam can be resolved. We further show that the optimum electronbeam scan speed is determined by a compromise between deflection of the cantilever at low scan speeds and limited spatial resolution at high scan speeds. Our technique will be an important tool for use in precise characterization of nanomechanical resonator devices. () 2013 AIP Publishing LLC. [http://dx.doi.org/10.1063/1.4811740]
\end{abstract}

\section{INTRODUCTION}

The mass resolution of a cantilever resonator mass sensor scales approximately as $k^{-0.5} M^{1.5}$ (Ref. 1), where $k$ is the stiffness and $M$ is the effective mass of the resonator. Nanoscale cantilevers, therefore, exhibit excellent mass resolution by comparison with the larger cantilevers typically used in scanning probe microscopy. Nanomechanical resonators have been proposed for a variety of applications including metal deposition monitors, mechanical reaction monitors, biomedical sensors, and mass sensors (Refs. 2-5). These sensors (Refs. 6 and 7) create opportunities for novel, labelfree detectors with high sensitivity and very high levels of multiplexing. While fabrication techniques for nanomechanical cantilevers are now rather mature (Refs. 8-13), measurement of the nanoscale displacement remains a challenge. For optimising the mass resolution, maximising the cantilever stiffness is also desirable, but this is accompanied by a decrease in the amplitude of the oscillations and so leads to a corresponding increase in transduction complexity. For micromechanical devices, transduction is achieved by detecting light from a laser beam which is reflected off the surface of the

\footnotetext{
a) Current address: Neue Materialien, Institut für Physik, HumboldtUniversität zu Berlin, Newtonstr.15, 12489 Berlin, Germany.

b) Author to whom correspondence should be addressed. Electronic mail: p.warburton@ucl.ac.uk.
}

cantilever. However, the reflected amplitude from a cantilever of sub-wavelength dimensions is vanishingly small. Alternative techniques are, therefore, being actively pursued for measurement of the vibration amplitude of cantilevers with submicron dimensions. Such techniques include capacitive readout (Ref. 14), piezoelectric read-out (Ref. 15), and hard electrical contact (Ref. 16).

The scanning-electron microscope (SEM) is a widely used tool for characterisation of the dynamic properties of nanomechanical devices. In undriven cantilevers, the cantilever resonant frequency has been determined by detecting oscillations at that frequency excited by either background mechanical noise (Ref. 12) or thermal fluctuations (Ref. 17). Alternatively, the cantilever may be driven at a frequency $f$ by (for example) a piezoelectric actuator inside the SEM vacuum chamber, and the cantilever oscillations are observed. This latter approach allows measurements of the full frequencyresponse of the resonator which additionally enables characterisation of the quality of the resonance.

For observing the cantilever oscillations, the SEM has typically been operated with the scan-deflection plates set at constant potential, commonly referred to as "spot mode," with two generic approaches described in the literature:

(i) To measure the resonant frequency $f_{0}$ and the quality factor $Q$ of the cantilever, the phase and amplitude of the secondary-electron signal are measured using lock-in detection at the drive frequency $f$ (Ref. 18). This technique 
does not, however, allow the extraction of the absolute magnitude of the displacement amplitude.

(ii) To determine the absolute displacement amplitude, $a$, of the cantilever, the primary electron beam is focussed at the equilibrium position. In the case when $a$ is much larger than the cantilever half-width $r$, the secondaryelectron signal is inversely proportional to the cantilever amplitude (Ref. 19).

As an alternative to this "spot-mode" method, Nonaka et al. (Ref. 17) introduced a technique based upon analysis of the secondary-electron signal from an electron beam which is linearly scanned across the oscillating resonator. This circumvents the limitations of the "spot-mode" approaches, in that it gives an absolute measurement of the amplitude $a$ even in the case that $a$ is less than the cantilever half-width $r$. Their method entails fitting the measured secondary-electron response to a model specific to the detailed material properties - in their particular case they assumed a cylindrical resonator with radially uniform material properties. In this paper we give a systematic description of a technique based on using a linearly scanned electron-beam. This technique is generically applicable and model-independent; it can, therefore, be straightforwardly applied to resonators with inhomogeneous materials properties and with arbitrary geometry. The accuracy with which the absolute amplitude can be measured in our approach is ultimately limited by the size of the focussed electron beam. Our method is likely to be important in the development of resonators with high stiffness (and hence high resonant frequency) for applications in high-resolution mass measurements. It is particularly suited to resonators of nanoscale cross-sectional dimensions in which the materials properties have not been well-characterised in advance.

\section{SECONDARY-ELECTRON RESPONSE FROM AN OSCILLATING CANTILEVER}

To provide a foundation for understanding the results we obtain with the experimental setup which we will describe in Sec. III, we begin by analysing the secondary-electron signal expected when an electron beam is incident upon a harmonically driven cantilever. The geometry is shown in Fig. 1: the cantilever, of width $2 r$, is oriented along the $y$-direction and oscillates in the $x y$ plane at a frequency $f$. The electron beam, which is oriented along an axis out of the $x y$ plane, is scanned continuously at a fixed value of $y$ across a length $L_{x}$ in the $x$-direction over a time $\tau_{x}$ at a constant speed $\mathrm{d} x / \mathrm{d} t$ $=L_{x} / \tau_{x}$. We denote the amplitude of the cantilever oscillation at this value of $y$ as $a$. If the electron-beam scan speed is much lower than the maximum speed of the cantilever (i.e., $\mathrm{d} x / \mathrm{d} t$ $\ll 2 \pi f a)$, then we may make the following quasistatic approximation: the secondary-electron signal at any position $x$ along the linescan is equal to that from a stationary electron beam at the same position.

The voltage generated by the secondary-electron detector is sampled at a rate $1 / \tau_{\mathrm{s}}$. To sample the oscillations of the cantilever adequately, and to maximise the signal-to-noise ratio, the measured voltage is obtained by integrating over $N \gg 1$ linescans, over a time $\tau_{\text {int }}$. We now define a response

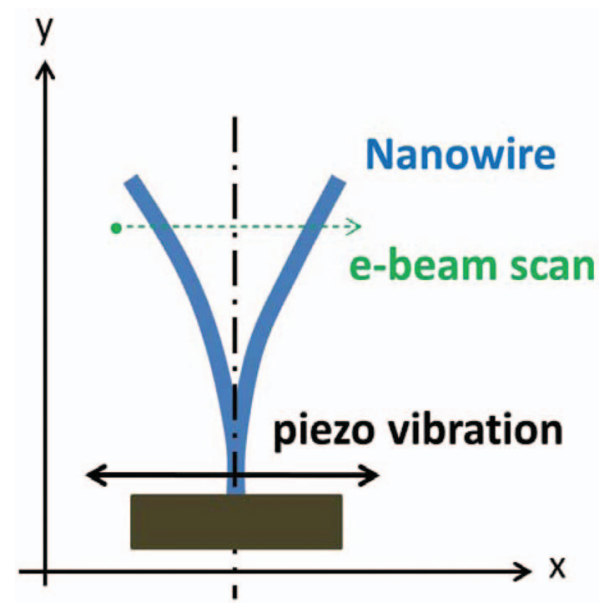

FIG. 1. Schematic diagram of the geometry of the electron beam and the nanowire. The nanowire oscillates in the $x y$ plane. The electron beam is oriented towards the $x y$ plane and scanned in the $x$ direction.

function $g(x)$ as the instantaneous secondary-electron (SE) voltage when the electron beam is scanned across the stationary cantilever. If the cantilever is driven with amplitude $a$ at frequency $f$, the instantaneous secondary-electron voltage is $g(x-a \sin \theta)$, where $\theta=2 \pi f t$. Since $N \gg 1$ and provided that $f \gg 1 / \tau_{\text {int }}$ and that $f$ is not commensurate with $1 / \tau_{\mathrm{s}}$ or $1 / \tau_{\text {linescan }}$, where $\tau_{\text {linescan }}$ is the time between the start of successive linescans, the measured signal will be the timeaveraged secondary-electron voltage over all possible positions of the nanowire:

$$
\left\langle V_{\mathrm{SE}}(x)\right\rangle=\frac{1}{2 \pi} \int_{0}^{2 \pi} g(x-a \sin \theta) d \theta .
$$

In principle, this allows the measured signal to be calculated for any arbitrary response function $g(x)$. It is important to note that $g(x)$ can be determined by measurements on the stationary cantilever. In the case where $g(x)$ is a "top hat" function (i.e., where $g(x)$ can take only two values: $g(x)=V_{\text {on }}$ when the electron beam is incident upon the cantilever and $g(x)$ $=V_{\text {off }}$ when it is not), $\left\langle V_{\mathrm{SE}}(x)\right\rangle$ can be calculated analytically as follows.

\section{A. Small amplitude, $a<r$}

First, we consider the case when the amplitude of the oscillations $a$ is less than the half-width of the cantilever, $r$, as shown in Fig. 2(a). For $|x|<(r-a)$ the electron beam hits the cantilever for all $\theta$, so the average secondary-electron voltage is $\left\langle V_{\mathrm{SE}}\right\rangle=V_{\text {on. }}$. For $|x|>(r+a)$, the electron beam misses the cantilever for all $\theta$, so the average secondary-electron voltage is $\left\langle V_{\mathrm{SE}}\right\rangle=V_{\text {off. }}$ For $(r-a)<|x|<(r+a)$, the e-beam hits the cantilever when $\theta_{1}<\theta<\theta_{2}$ (as shown by the horizontal dashed line in Fig. 2(a)) and misses the cantilever for the rest of the full cycle. Hence,

$$
\left\langle V_{\mathrm{SE}}\right\rangle=\frac{1}{2 \pi}\left\{V_{\text {on }}\left(\theta_{2}-\theta_{1}\right)+V_{\text {off }}\left(2 \pi-\left(\theta_{2}-\theta_{1}\right)\right)\right\},
$$



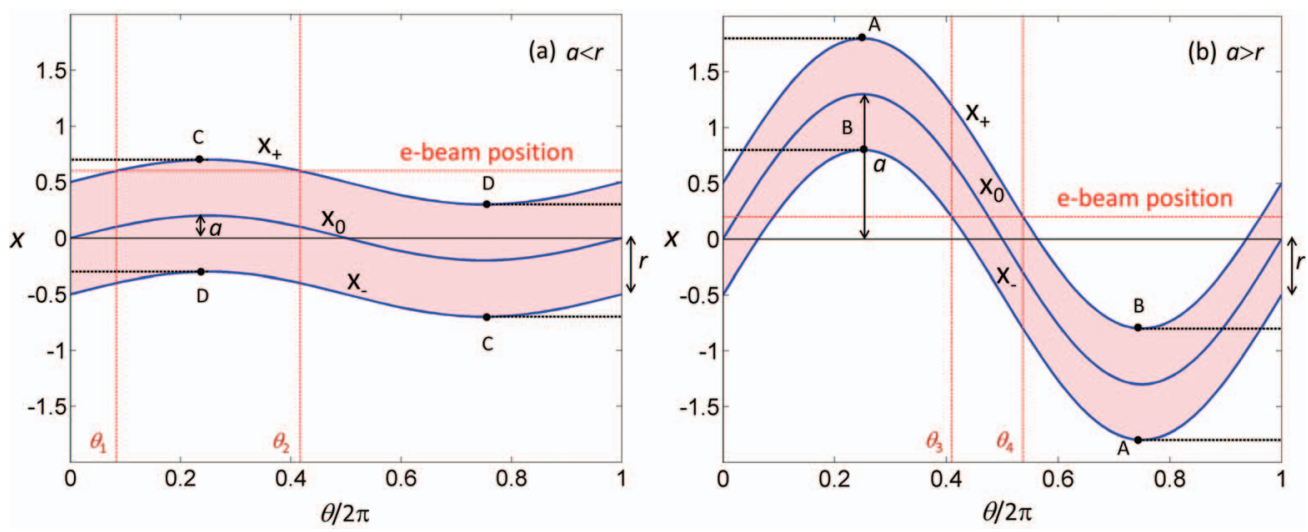

FIG. 2. The position of the central axis $x_{0}$ and edges $x_{+}$and $x_{-}$of a cantilever of width $2 r$ as a function of a time coordinate $\theta=2 \pi f t$. In (a) the amplitude $a$ of the oscillation is smaller than $r$; the horizontal dashed line shows an illustrative electron-beam scan for the case $(r-a)<|x|<(r+a)$. In (b) the amplitude $a$ of the oscillation is larger than $r$; the horizontal dashed line shows an illustrative electron-beam scan for the case $|x|<(a-r)$.

where $\theta_{1}=\sin ^{-1}\{(x-r) / a\}$ and $\theta_{2}=\pi-\theta_{1}$. Therefore, defining a normalised voltage, $v_{N}$ :

$$
\begin{array}{r}
v_{N} \equiv \frac{\left\langle V_{\mathrm{SE}}\right\rangle-V_{\text {off }}}{V_{\text {on }}-V_{\text {off }}}=\frac{1}{2 \pi}\left[\pi-2 \sin ^{-1}\left(\frac{|x|-r}{a}\right)\right] \\
{[a<r,(r-a)<|x|<(r+a)] .}
\end{array}
$$

An illustration of the dependence of the resulting timeaveraged secondary-electron signal upon the electron-beam position $x$ is shown in Fig. 3 for the case $a / r=0.4$. There is a discontinuity in the gradient at two (pairs of) positions of the electron beam. The corresponding $x$ positions at which these discontinuities occur are marked in Fig. 2, and the distance

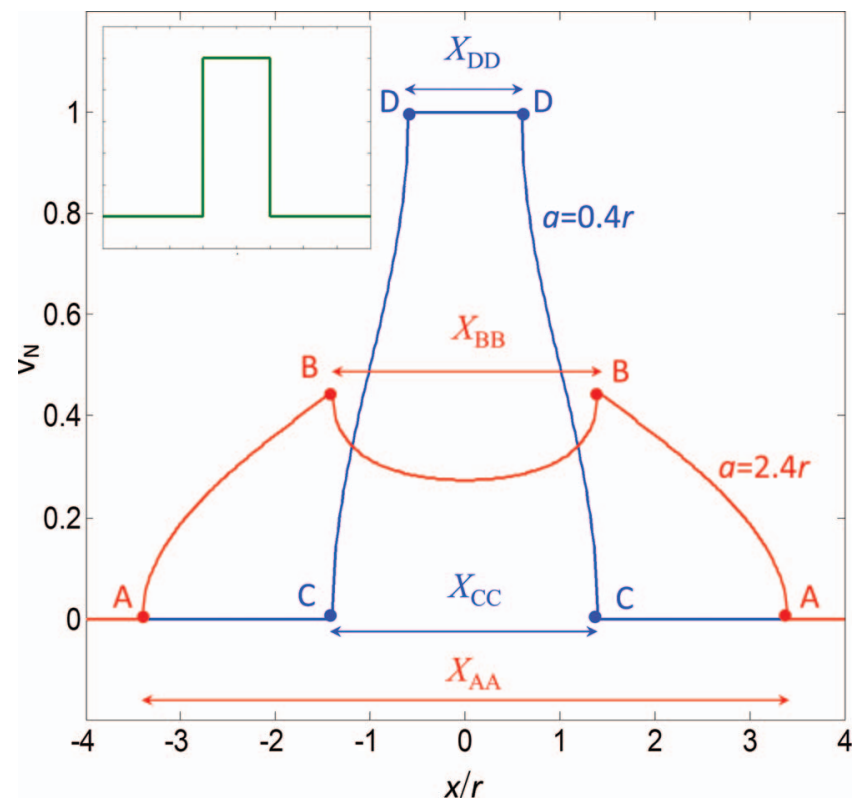

FIG. 3. Calculated time-averaged normalised secondary-electron voltage as the electron beam is scanned across a cantilever of width $2 r$ oscillating with amplitude $a$. The inset shows, to the same scales, the assumed response of a stationary cantilever - a top-hat function. Blue line: $a=0.4 r$; red line: $a=$ $2.4 r . X_{\mathrm{AA}}, X_{\mathrm{BB}}, X_{\mathrm{CC}}$, and $X_{\mathrm{DD}}$ are the distances between pairs of points $\mathrm{A}$, $\mathrm{B}, \mathrm{C}$, and $\mathrm{D}$, respectively, which mark gradient discontinuities in the voltage (see text for details). between these two positions is independent of $r$ :

$$
X_{C C}-X_{D D}=4 a,
$$

and can, therefore, be used experimentally to determine the vibration amplitude.

\section{B. Large amplitude, $a>r$}

We now turn to the case when the amplitude of the oscillations $a$ is greater than the half-width of the cantilever, $r$, as shown in Fig. 2(b). For $|x|>(r+a)$, the electron beam misses the cantilever for all $\theta$, so the average secondary-electron voltage is $\left\langle V_{\mathrm{SE}}\right\rangle=V_{\text {off. }}$. For $(a-r)<|x|<(a+r)$, the analysis is identical to the case when $a<r$ and $(r-a)<|x|<(r$ $+a$ ) discussed above and the SE voltage can be determined by Eq. (3).

For $|x|<(a-r)$, the e-beam hits the cantilever when $\theta_{3}$ $<\theta<\theta_{4}$ (as shown by the horizontal dashed line in Fig. 2(b)) and misses the cantilever for the rest of the half-cycle:

$$
\left\langle V_{\mathrm{SE}}\right\rangle=\frac{1}{\pi}\left\{V_{o n}\left(\theta_{4}-\theta_{3}\right)+V_{o f f}\left(\pi-\left(\theta_{4}-\theta_{3}\right)\right)\right\},
$$

where $\theta_{3}=\sin ^{-1}\{(x-r) / a\}$ and $\theta_{4}=\sin ^{-1}\{(x+r) / a\}$, giving

$$
\begin{array}{r}
v_{N}=\frac{1}{\pi}\left[\sin ^{-1}\left(\frac{x+r}{a}\right)-\sin ^{-1}\left(\frac{x-r}{a}\right)\right] \\
{[a>r,|x|<(a-r)] .}
\end{array}
$$

An illustration of the resulting time-averaged secondaryelectron signal is shown in Fig. 3 for the case $a / r=2.4$. As in the case for $a<r$, the separation between the gradient discontinuities in $\left\langle V_{\mathrm{SE}}(x)\right\rangle$ may be used to determine the amplitude, this time through the relation $a=\left(X_{\mathrm{AA}}+X_{\mathrm{BB}}\right) / 4$.

Figure 4 shows the calculated time-averaged secondaryelectron voltage as a function of position across a cantilever of width $2 r$ as the amplitude $a$ increases from 0 to $2.8 r$, assuming a "top-hat" response function $g(x)$. Note that, provided the amplitude is small enough (i.e., $a \leq 2 r$ ), all the linescans pass through the points $|x|=r, v_{N}=0.5$. 


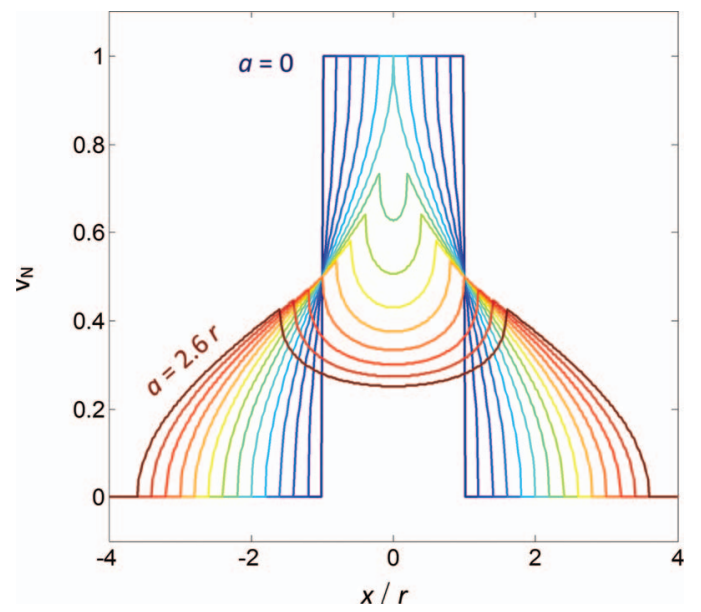

FIG. 4. Calculated time-averaged normalised secondary-electron voltage as the electron-beam is scanned across a cantilever of width $2 r$. The oscillation amplitude $a$ varies from 0 to $2.6 r$ in steps of $0.2 r$. The static response is assumed to be a top-hat function (see the $a=0$ curve).

Note also that at $x=0$ there is a local minimum given by

$$
v_{N}=\frac{2}{\pi} \sin ^{-1}\left(\frac{r}{a}\right) .
$$

In the limit $a \gg r$, this approximates to

$$
v_{N}=\frac{2 r}{\pi a} .
$$

Hence, the inverse of the time-averaged secondary-electron signal at the equilibrium position $x=0$ in the limit of large amplitude oscillations is a direct measure of the oscillation amplitude. This was noted previously by Nishio et al. (Ref. 19) in the context of carbon nanotube mechanical resonators.

\section{EXPERIMENTAL SETUP}

Our experimental configuration for measuring the frequency response of nanomechanical resonators in a SEM is

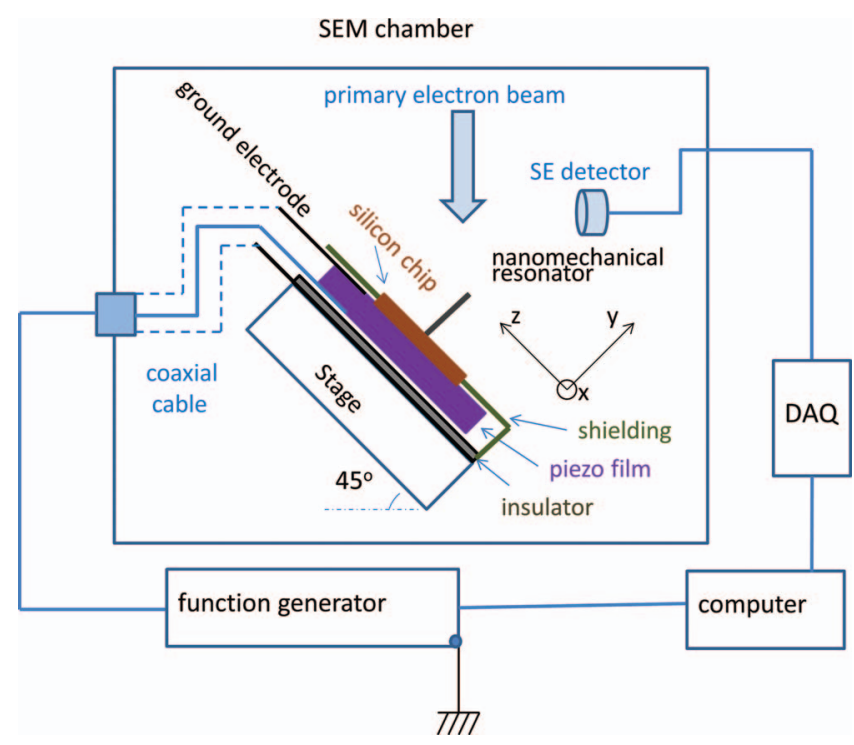

FIG. 5. Schematic diagram of the experimental setup. (SE: secondaryelectron; DAQ: data acquisition card.)

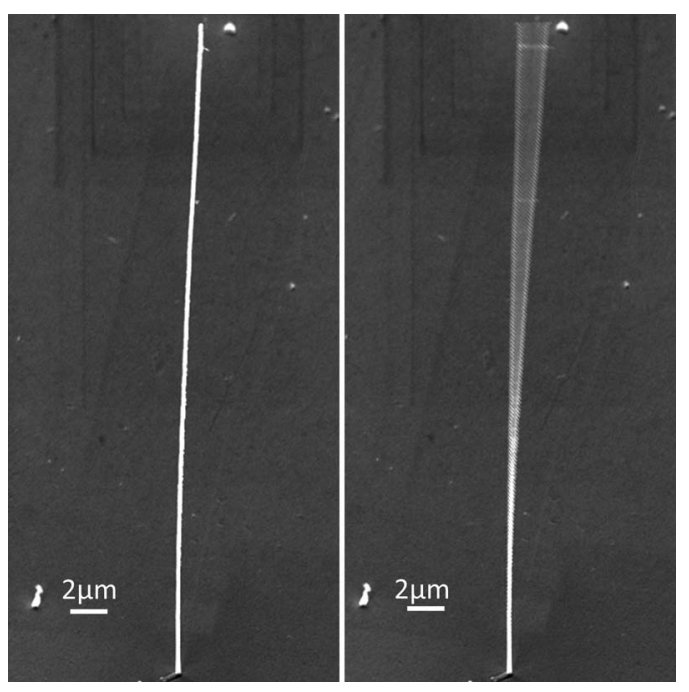

FIG. 6. SEM images of a nanowire of length $58 \mu \mathrm{m}$, (a) nominally stationary, and (b) driven by the piezoelectric actuator at its resonant frequency (here $25.6 \mathrm{kHz}$ ).

shown in Fig. 5. The resonator is mounted on a piezoelectric film which is oriented at an angle of $45^{\circ}$ with respect to the primary electron beam. The piezoelectric film is polyvinylidene fluoride (PVDF) with thin-film gold electrodes. The primary electron beam is oriented along an axis out of the cantilever oscillation ( $x y$ ) plane (Fig. 1); due to geometrical constraints, this is not perpendicular to the oscillation plane. The electron-beam energy was typically $10 \mathrm{keV}$. A digital-toanalogue converter (National Instruments PCI 6035E) with a maximum sampling rate of 390000 samples/s was used to acquire the real-time signal from the secondary-electron detector. The secondary-electron response from 256 linescans was collected in order to ensure adequate sampling in forming the average response at each position. To satisfy the condition $f \gg 1 / \tau_{\text {int }}$, discussed above, measurements were restricted to $f>\sim 3 \mathrm{kHz}$.

In order to test our measurement technique, we have grown nanowires using focussed-ion-beam-assisted deposition. The nanowires are deposited on a silicon substrate using a Carl Zeiss XB1540 FIB system operated in spot mode so that the nanowires grow vertically, and tungsten hexacarbonyl $\left[\mathrm{W}(\mathrm{CO})_{6}\right]$ was used as a precursor gas (Ref. 20). The gallium ion beam energy and current were $30 \mathrm{keV}$ and $1 \mathrm{pA}$, respectively. The nanowires typically have a length between 20 and $60 \mu \mathrm{m}$ and a radius between 60 and $140 \mathrm{~nm}$. Figure 6(a) shows an electron micrograph of a static nanowire of length $58 \mu \mathrm{m}$. Figure 6(b) shows the same nanowire being harmonically driven by the piezoelectric film.

\section{EXPERIMENTAL RESULTS AND DISCUSSION}

Figure 7 shows two experimentally measured linescans of oscillating cantilevers, one with smaller-amplitude vibrations and one with larger-amplitude vibrations. Comparison to the analytical results shown in Fig. 3 shows very good qualitative agreement. This agreement is further seen by comparison of the analytical results shown in Fig. 4 with Fig. 8, which shows more detailed measurements in which the 


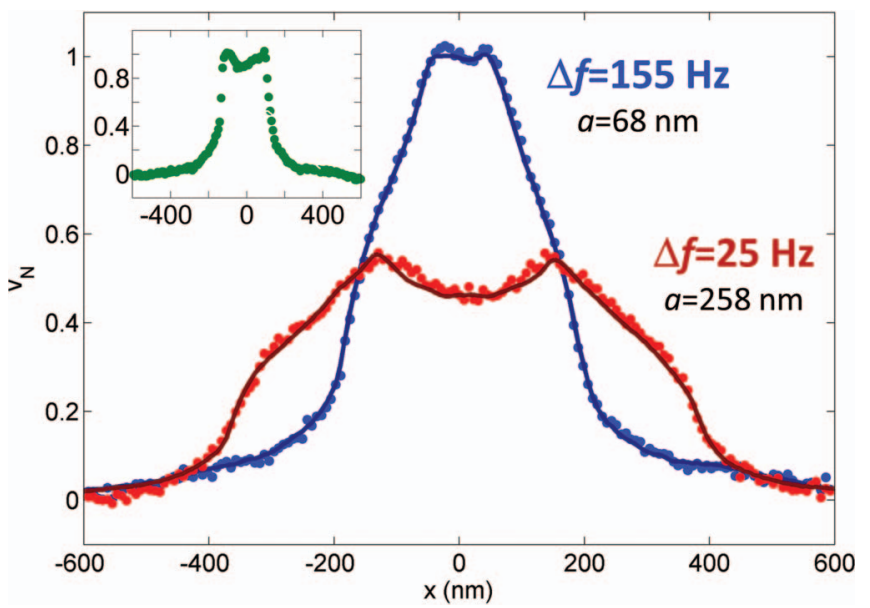

FIG. 7. Measured secondary-electron response as a function of $x$ position across a vibrating nanowire of diameter $233 \mathrm{~nm}$, length $61 \mu \mathrm{m}$, and resonant frequency $26.55 \mathrm{kHz}$. Blue points: nanowire driven at $26.70 \mathrm{kHz}$. Red points: nanowire driven at $26.59 \mathrm{kHz}$. The detuning $\Delta f \equiv f-f_{0}$ is indicated. The inset shows, to the same scales, the stationary response of the nanowire. The lines in the main plot show fits to Eq. (1) using this stationary response, as described in the text, and the corresponding fitted amplitude is indicated.

driving frequency is swept towards the resonant frequency of the cantilever, thereby changing the amplitude of the cantilever oscillations. Due to the stiffness of this cantilever, the amplitude at resonance is not significantly larger than its radius. The good qualitative agreement with the calculations is shown, for example, in the clear reproduction in the experimental data of the predicted intersection of all the linescans at $|x|=r$, at a normalised secondary-electron voltage of 0.5. There are some quantitative differences, however. The gradient discontinuities are somewhat less marked in the experimental data; this is associated with the measured signal from a (nominally) stationary cantilever departing from the "top-hat" function we assumed in Sec. II. We also observed that the measured secondary-electron signal from an oscillating cantilever depends upon the speed $\mathrm{d} x / \mathrm{d} t$ at which the

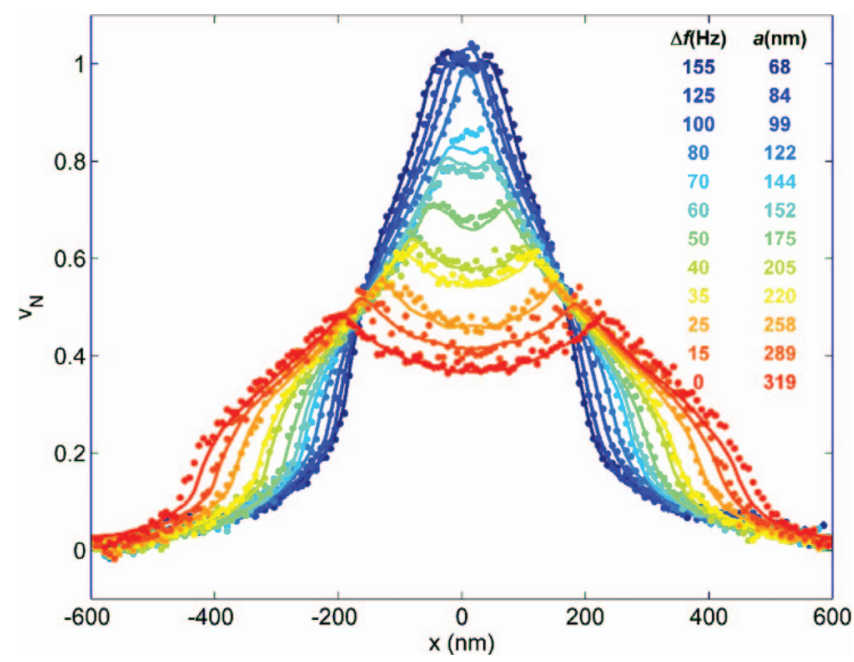

FIG. 8. Measured secondary-electron response as a function of frequency, for the same resonator as shown in Fig. 7. The detuning and fitted value of the oscillation amplitude are indicated in the legend. primary electron beam scans across it. We now discuss how each of these features impacts upon the interpretation of the measurements.

\section{A. Static response function}

The secondary-electron signal measured during a linescan with no voltage applied to the piezoelectric actuator has an appreciably more rounded form than for the "top-hat" function used in the analysis in Sec. II. The inset of Fig. 7 shows one such measurement. The difference between the shapes is caused by a number of factors. Since the nanowires are circular in cross section, secondary electrons generated near $x= \pm r$ are on average more likely to escape from the nanowire than those generated near $x=0$. This leads to an increase in the secondary electron response near $x= \pm r$ as described in Nonaka et al. (Ref. 17). In addition, even in the absence of any oscillating potential applied to the piezoelectric actuator, the nanowires display small oscillations at the resonant frequency which are excited by background mechanical noise and/or thermal fluctuations. This broadens the edges of the measured "stationary" response.

\section{B. Driven response: Extraction of oscillation amplitude and its resolution}

Figure 7 shows the response of the cantilever when driven at two frequencies close to its resonant frequency. Since the static-response function is rounded (Fig. 7, inset) we cannot straightforwardly extract absolute measurements of the oscillation amplitude using the separation of the gradient discontinuities (Sec. II B and Fig. 3), but instead extract the amplitude by numerically fitting the measured dynamic response function to Eq. (1), using the measured static response $g(x)$. The amplitude $a$ is used as a fitting parameter. The black lines in

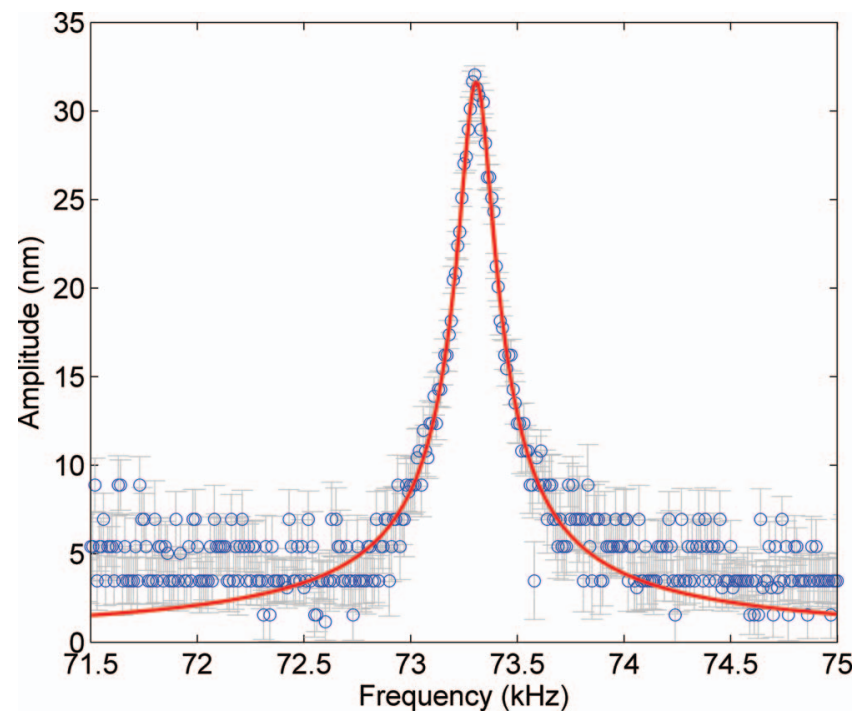

FIG. 9. Frequency response of a resonator with length $43 \mu \mathrm{m}$ and diameter $275 \mathrm{~nm}$. The blue points are extracted from the experimental measurements as described in the text and the red line is the best Lorentzian fit to the data in the range $73.04-73.63 \mathrm{kHz}$, corresponding to a resonant frequency of $73.31 \mathrm{kHz}$ and a $Q$-factor of 425 . 

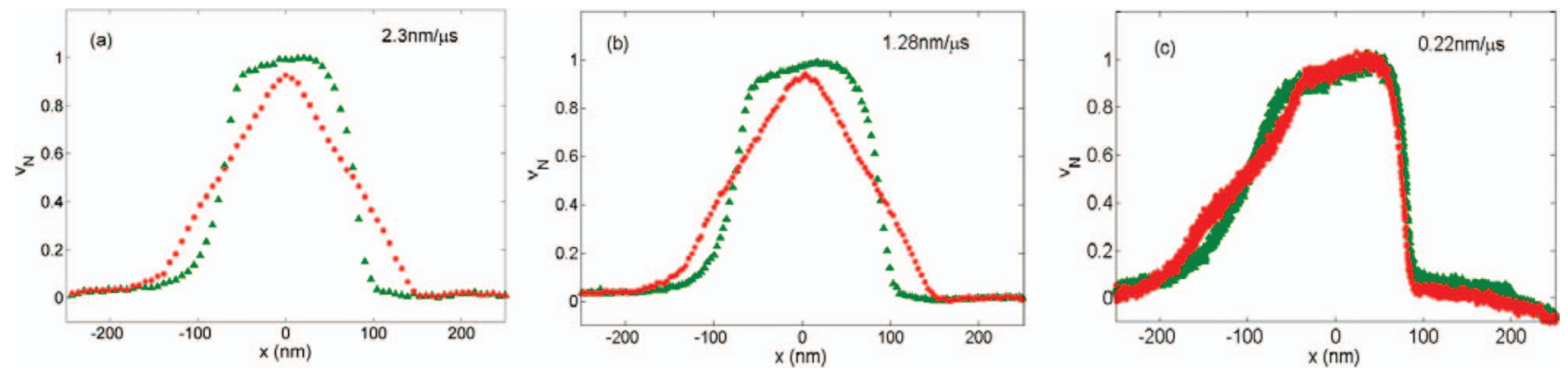

FIG. 10. Scan-speed dependence of linescans across a nanowire of length $25 \mu \mathrm{m}$ and diameter $120 \mathrm{~nm}$ with resonant frequency $94.96 \mathrm{kHz}$. Green triangles correspond to when the resonator is nominally stationary; red circles to when it is driven at a frequency of $95.04 \mathrm{kHz}$, a detuning of $80 \mathrm{~Hz}$. Departures from desirable behaviour in (a) and (c) are described in the text.

Fig. 7 show the least-squares best fits to the measured dynamic responses using this method. The fit gives an excellent match to the experimental data (Ref. 21). Note that the fit is good across the whole range of $x$ and that the fitting procedure is independent of any model of how electrons interact with the nanowire, so it was not necessary to account for changes in these interactions across the wire associated with the likely gallium-rich core of the nanowire (Ref. 17).

By fitting the measured response of the cantilever over a range of drive frequencies (shown in Fig. 8) to Eq. (1) using the measured static response, we extract the vibration amplitude as a function of the driving frequency; this is shown in Fig. 9. There is a clear peak and the vibration amplitude is well fitted by a Lorentzian. The extracted resonant frequency of $73.31 \mathrm{kHz}$ agrees reasonably well with the value of $79.5 \mathrm{kHz}$ calculated using the theory of bending mechanics, assuming circular cross section and representative values for the density and Young's modulus of the FIB-deposited tungsten (Ref. 22). Away from the resonant peak, the noise floor of the technique is reached. The amplitude value at the noise floor decreases as $N$ increases. For our measurements, with $\mathrm{N}=256$, shown in Fig. 9, the minimum resolvable oscillation amplitude is $\approx 8 \mathrm{~nm}$.

It is worth noting that the presence of oscillations in the stationary response (see Sec. IV A) does not affect the validity of our fitting procedure - the extracted amplitude represents the amplitude of oscillations additional to those present in the "stationary" response (Ref. 23). In particular, the amplitude of driven oscillations may be measured even in the presence of (perhaps much larger) low-frequency mechanical oscillations.

In principle, using our technique the minimum resolvable amplitude is ultimately limited to amplitudes similar to the lengthscale over which the steepest changes in secondary electron signal occur for a stationary cantilever - related to the size of the electron beam in the case of a truly stationary cantilever. A second limitation is that features on lengthscales smaller than the step size $\tau_{\mathrm{s}} \mathrm{d} x / \mathrm{d} t$ in the linescan data are not resolved and this affects, in particular, the fits to smallamplitude oscillations such as those seen at the edges of the peak in Fig. 9. This step size can be decreased by decreasing the beam speed - although not to arbitrarily smaller values, as discussed in Sec. IV C - or by increasing the sampling frequency. Thus, the intrinsic resolution of the technique could be reached by using a larger sampling frequency than that used for Fig. 9.

\section{Effect of electron-beam scanning speed}

There is a range of scanning speeds for which the secondary-electron signal provides data matched well by the model we have described in Sec. II. The measurements shown in Figs.7-9 were all made using $\mathrm{d} x / \mathrm{d} t \sim 2 \mathrm{~nm} / \mu \mathrm{s}$. In fact, it is important to choose the scan speed carefully. Figure 10 shows the secondary-electron signal as the primary electron beam is scanned in the $x$-direction at different constant scanning speeds $\mathrm{d} x / \mathrm{d} t$ across a cantilever with resonant frequency $94.96 \mathrm{kHz}$. For the green curves, the cantilever is nominally stationary (i.e., the voltage across the piezoelectric actuator stage is zero); for the red curves, the cantilever is driven by applying a sinusoidal voltage of amplitude $20 \mathrm{~V}$ and frequency $95.04 \mathrm{kHz}$ to the piezoelectric actuator. At low scanning speeds, there is a marked asymmetry in the measured secondary-electron signal. This asymmetry results from beam-induced deflection of the nanowire. On the other hand, at very high scanning speeds when $\tau_{\mathrm{s}} \mathrm{d} x / \mathrm{d} t \ll a$ breaks down, the finite maximum sampling rate results in a loss of spatial resolution. We find that an optimum compromise between these two effects is obtained with a scanning speed of around $2 \mathrm{~nm} / \mu \mathrm{s}$; this gives negligible beam-induced deflection and a measurement spacing $(1 \mathrm{~nm})$ which is less than the electronbeam full width at half maximum $(\sim 1.5 \mathrm{~nm})$. For amplitudes and frequencies reported here, this scan speed keeps our measurements in the quasi-static limit, i.e., $\mathrm{d} x / \mathrm{d} t$ is an order of magnitude smaller than $2 \pi f a$ as discussed in Sec. II.

\section{v. CONCLUSIONS}

We have experimentally demonstrated that, by performing electron linescans of oscillating cantilevers in a scanningelectron microscope, it is possible to measure absolute nanoscale displacements of the oscillation amplitude even when the displacement is significantly less than the cantilever radius. No prior knowledge of the cantilever geometry is required, nor need its materials properties be radially homogeneous. The resolution of our technique is likely to prove useful for detailed investigations of the dynamics of nanomechanical resonator vibration, for the more accurate modelling of these systems through the determination of realistic boundary conditions and homogeneity of the mechanical properties. Since maximising the mass resolution of a nanomechanical mass balance by maximising its stiffness also means a decrease in 
the amplitude of the oscillations, this technique is likely to be of particular significance for calibrating displacements of nanomechanical resonators with high stiffness and, therefore, small amplitude. This SEM linescan method will be a useful tool in the development of nanomechanical resonators with high stiffness (for example, nanoscale silicon cantilevers) for applications in mass sensing.

\section{ACKNOWLEDGMENTS}

We thank Dr. Suguo Huo for technical assistance. This work is supported by the Engineering and Physical Sciences Research Council (United Kingdom) (EPSRC).

${ }^{1}$ K. L. Ekinci, Y. T. Yang, and M. L. Roukes, J. Appl. Phys. 95, 2682 (2004).

${ }^{2}$ H. J. Mamin and D. Rugar, Appl. Phys. Lett. 79, 3358 (2001).

${ }^{3}$ B. Ilic, D. Czaplewski, M. Zalalutdinov, H. G. Craighead, P. Neuzil, C. Campagnolo, and C. Batt, J. Vac. Sci. Technol. B 19, 2825 (2001).

${ }^{4}$ A. Gupta, D. Akin, and R. Bashir, J. Vac. Sci. Technol. B 22, 2785 (2004).

${ }^{5}$ B. Ilic, D. Czaplewski, H. G. Craighead, P. Neuzil, C. Campagnolo, and C. Batt, Appl. Phys. Lett. 77, 450 (2000).

${ }^{6}$ H. G. Craighead, Science 290, 1532 (2000).

${ }^{7}$ K. L. Ekinci and M. L. Roukes, Rev. Sci. Instrum. 76, 061101 (2005).

${ }^{8}$ D. W. Carr, L. Sekaric, and H. G. Craighead, J. Vac. Sci. Technol. B 16, 3821 (1998)

${ }^{9}$ S. S. Verbridge, J. M. Parpia, R. B. Reichenbach, L. M. Bellan, and H. G. Craighead, J. Appl. Phys. 99, 124304 (2006).
${ }^{10}$ A. Husain, J. Hone, H. W. C. Postma, X. M. H. Huang, T. Drake, M. Barbic, A. Scherer, and M. L. Roukes, Appl. Phys. Lett. 83, 1240 (2003).

${ }^{11}$ S. Evoy, M. A. Riegelman, N. Naguib, Y. Haihui, P. Jaroenapibal, D. E. Luzzi, and Y. Gogotsi, IEEE Trans. Nanotechnol. 4, 570 (2005).

${ }^{12}$ J. Fujita, M. Ishida, T. Sakamoto, Y. Ochiai, T. Kaito, and S. Matsui, J. Vac. Sci. Technol. B 19, 2834 (2001)

${ }^{13}$ V. Sazonova, Y. Yaish, H. Ustunel, D. Roundy, T. A. Arias, and P. L. McEuen, Nature (London) 431, 284 (2004)

${ }^{14}$ S. Ghatnekar-Nilsson, I. Karlsson, A. Kvennefors, G. Luo, V. Zela, M. Arlelid, T. Parker, L. Montelius, and A. Litwin, Nanotechnology 20, 175502 (2009).

${ }^{15}$ V. Friedli, C. Santschi, J. Michler, P. Hoffman, and I. Utke, Appl. Phys. Lett. 90, 053106 (2007).

${ }^{16}$ S. Dohn, O. Hansen, and A. Boisen, Appl. Phys. Lett. 88, 264104 (2006).

${ }^{17}$ K. Nonaka, K. Tamaru, M. Nagase, H. Yamaguchi, S. Warisawa, and S. Ishihara, Jpn. J. Appl. Phys. 48, 06FG07 (2009).

${ }^{18}$ V. Friedli, I. Utke, K. Molhave, and J. Michler, Nanotechnology 20, 385304 (2009).

${ }^{19}$ M. Nishio, S. Sawaya, S. Akita, and Y. Nakayama, Appl. Phys. Lett. 86, 133111 (2005).

${ }^{20}$ W. X. Li and P. A. Warburton, Nanotechnology 18, 485305 (2007).

${ }^{21}$ The fit was conducted over the range $|x|<\left(L_{x} / 2-a\right)$ - for which $g(x)$ was measured for all $\theta$. In Figs. 7 and 8, the fit lines are extended to cover the whole range of the experimental data, with additional values added by extending the range of the function $g(x)$ and assuming it to remain constant beyond its measured range.

${ }^{22}$ H. Wang, Ph.D. dissertation, University College London, 2013.

${ }^{23}$ This is for the generally applicable case when the additional oscillations are not commensurate with the oscillations broadening the stationary response. For the special case where the resonator is driven at its resonant frequency, the fitted amplitude gives $\sqrt{ }\left(a_{\mathrm{d}}{ }^{2}-a_{\mathrm{u}}{ }^{2}\right)$, where $a_{\mathrm{d}}$ is the amplitude of driven oscillations at the resonant frequency and $a_{\mathrm{u}}$ is the amplitude of undriven (e.g., thermally excited) oscillations at the resonant frequency. 